\title{
Correction to "CCAAT/Enhancer-Binding Protein $\beta$ (Nuclear Factor for Interleukin 6) Transactivates the Human MDR1 Gene by Interaction with an Inverted CCAAT Box in Human Cancer Cells"
}

\author{
In the above article [Chen GK, Sale S, Tan T, Emoian RP, and Sikic BI (2004) Mol \\ Pharmacol 65: 906-916 DOI: https://doi.org/10.1124/mol.65.4.906], the first and middle \\ name of the author G. Kevin Chen needs to be corrected to Kevin G. Chen.
}

The authors regret any inconvenience it may have caused. 